\title{
A Knowledge Based Framework to Support Active Aging at Home Based Environments
}

\author{
Miguel Ángel Valero , José Bravo , Juan Manuel García , \\ Diego López-de-Ipiña, and Ana Gómez
}

\begin{abstract}
Information and Communication Technologies can support Active Aging strategies in a scenario like the Smart Home. This paper details a person centered distributed framework, called TALISMAN+, whose aim is to promote personal autonomy by taking advantage of knowledge based technologies, sensors networks, mobile devices and internet. The proposed solution can support an elderly person to keep living alone at his house without being obliged to move to a residential center. The framework is composed by five subsystems: a reasoning module that is able to take local decisions at home in order to support active aging, a biomedical variables telemonitorisation platform running on a mobile device, a hybrid reasoning middleware aimed to assess cardiovascular risk in a remote way, a private vision based sensor subsystem, and a secure telematics solution that guarantees confidentiality for personal information. TALISMAN+ framework deployment is being evaluated at a real environment like the Accessible Digital Home.
\end{abstract}

\section{Framework Contextualization}

Active Aging is not just a set of recommendations for physical and psychological well-being but a "process of optimizing opportunities for health, participation and security in order to enhance quality of life as people age" [1]. The World Health Organization (WHO) highlights the necessity to promote effective strategies and solutions that maintain autonomy as a person grows older. Many daily life activities are carried out at the home environment and this user domain is widely surrounded by multiple devices and appliances which are supposed to make our life "easier".

The interoperation at home of these available mechanic, electronic, information and communication technologies, sets the basis to provide the elderly population 
and/or people with neurodegenerative diseases with accessible services to promote personal autonomy. Cheek et al. mention the concept of aging-in-place and point out different facilities to be supported by Smart Home ( $\mathrm{SH}$ ) technologies such as "emergency care, fall prevention \& detection, reminder systems and assistance for those with cognitive impairments" [2]. This idea is not new, some authors like Williams et al. described in 1998 their future smart home for "the provision of artificial intelligence -AI- based information processing and the management of decision-making structures required" [3]. Fifteen years later, users could request for SH technologies at their house since environmental sensors like presence, motion, fire, flood or gas are market available; biomedical data devices can be connected to measure pulse, temperature, glucose or blood pressure, and reasoning middleware environments are highly usable. However, the easy to use and knowledge based person centered interaction with these devices is still not solved especially to support aforementioned SH facilities. TALISMAN+, the distributed framework detailed in this research paper, aims to go one step forward in this direction by offering an integrated solution that encompasses local and remote reasoning modules, biomedical mobile connected telemonitoring devices, environmental sensor networks and security mechanisms that guarantee privacy and confidentiality of supported homecare telematic services. The framework core has been designed and developed by taking into account the necessities of two users domains: vulnerable people with Parkinson disease and persons with mobility restrictions.

Information and communication technologies can help people with cognitive and mobility impairments to promote Active Aging activities related to communication, stimulation and environmental control. Laiseca et al. showed the utilization of these technologies to assist the elderlies with cognitive disabilities by using memory games that facilitate information to caregivers and relatives [4]. Activity recognition can be triggered both from data driven information provided by users or through the utilization of sensor-based recognition. Chen et al. compare these two approaches and conclude that Knowledge-Driven models need to handle uncertainty and time in order to distinguish intent or goal recognition [5]. Ontology-based systems have been tested to support active health with mobile technologies. Docksteader et al. published a Mobile Ontology-based Reasoning and Feedback system that monitors SpO2, using Semantic Web Rule Language (SWRL) and communicate them via SMS and HTTP protocols [6]. Health care domain has also experimented with the use of cameras in private spaces in the field of Ambient-Assisted Living (AAL) and aging in place. Cardinaux et al. reviewed in 2011 the pros and contras, related to user's acceptance, reliable reasoning and privacy, of video based technology for AAL [7].

No doubt that TALISMAN+ framework provides facilities that can be critical for the security of the elderly. Therefore, privacy of monitored individuals should be guaranteed at the same time its identities are checked as Islam et al. states for SH [8]. Since sensors and devices used can be perceived as an intrusive element at home [12], privacy and authentication issues were considered an essential part of this framework in order to reinforce the trust of users to promote their personal autonomy. 


\section{User Driven Design Methodology}

Further to ANSI/IEEE 1471-2000 conceptual framework for architecture description, TALISMAN+ design can be decomposed in five architectural views. The methods provided by this standard helped to describe the global view of the solution according to a user driven approach. Thus, people with cognitive or mobility restrictions, such as the elderly, become the main stakeholder addressed by each of the five deployed subsystems. The mission is to support autonomous active aging at home by providing context-aware reactions triggered by detected events, user profiles and reasoning procedures. Main concerns addressed in this framework deal with security, accessibility, reliable reasoning and interoperability. Security view followed a user centered approach so that an aging person may feel trust about his or her interaction with the system. Therefore, user requirements analysis led to define a user interface so that all the stakeholders may simulate, check and effectively validate the suggestions provided by the framework. These stakeholders include the elderly person, informal caregivers, relatives, and professionals in charge such us geriatricians, therapists, social workers or nurses.

By following a user driven design methodology, replicable user cases were defined according to the knowledge acquired from two users' entities: Madrid Parkinson's disease association and the association of people with spinal injury and physical disabilities (ASPAYM). As Gass et al. state for internet-based services, the specification of end-user-driven data acquirement at the $\mathrm{SH}$ was a critical issue to define context-aware interoperable facilities to promote active aging [9].

Fig. 1 details the functional Framework design whose user driven main use case is detailed as follow: 1) Biomedical data provided by users through mobile connected sensors is sent to the reasoning subsystems; 2 ) the hybrid remote reasoner validates a user profile (e.g. level of risk disease) and sends this data to the local reasoner; 3 ) environmental context and user profile info is updated to the local reasoner; 4) a descriptive local reasoner suggests actions at the SH for active aging; 5) the security view ensures authentication, confidentiality and integrity of managed information.

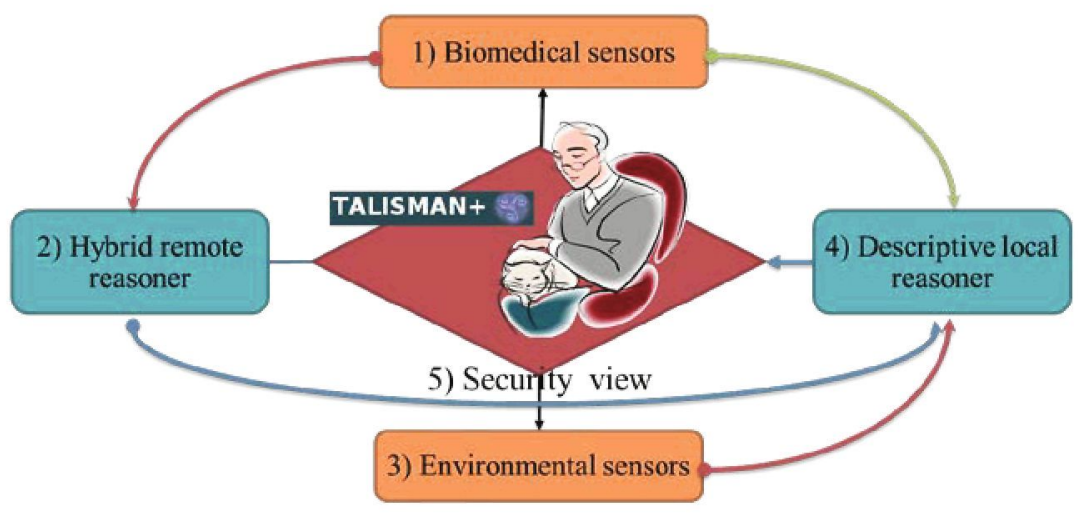

Fig. 1. User-driven functional Framework Design 


\section{Development Results}

The resulting distributed framework is described in the following parts by following the user-driven context previously depicted. :

\subsection{Mobile Monitoring (MoMo) Platform}

The Mobile Monitoring platform (MoMo) allows aging patients to having continuous diseases control and direct communication with their doctor. MoMo enables patient mobile telemonitoring by using biometric devices (e.g. glucometers, blood pressure meters) to send data to a mobile phone via technologies such as WiFi, NFC or Bluetooth [10]. An ontological architecture has been created in order to catalogue the elements and provide TALISMAN+ local and remote reasoners with ad hoc feedback.

Patient monitoring represents one of the key elements in the progress and control of his illness. This monitoring provides patient and doctor with continuous data about disease's status (vital signs, pulse glucose) so that, the doctor can accordingly readjust the initial treatments and prescriptions. Mobile phone is the selected technology as it is fairly used by aging people and can support daily activities for communication and information management. A group of ontologies called MoMOntology represent the ontologies of mobile monitoring process and allow to model the data collected from biometrics devices. An analytical engine, described in 3.2 which combines Fuzzy Logic and probabilistic reasoning, allows managing patient records based on an analysis of past situations to predict future difficulties like variations in vital signs). MoMo takes advantage of mobile phones and biometric devices to facilitate patient monitoring as data is recorded in a central server to be used by TALISMAN+ framework.

Fig. 2 shows the developed platform. On the left, healthcare and monitoring devices are connected to Bluetooth mobile devices. In the center, these biometric devices are linked to a mobile phone to process sensor data, manage applications, and ensure redundant connectivity via $3 \mathrm{G}$ and $\mathrm{WiFi}$ data networks. Information is transmitted to a central database and advisory system for evaluation by the medical server (right).

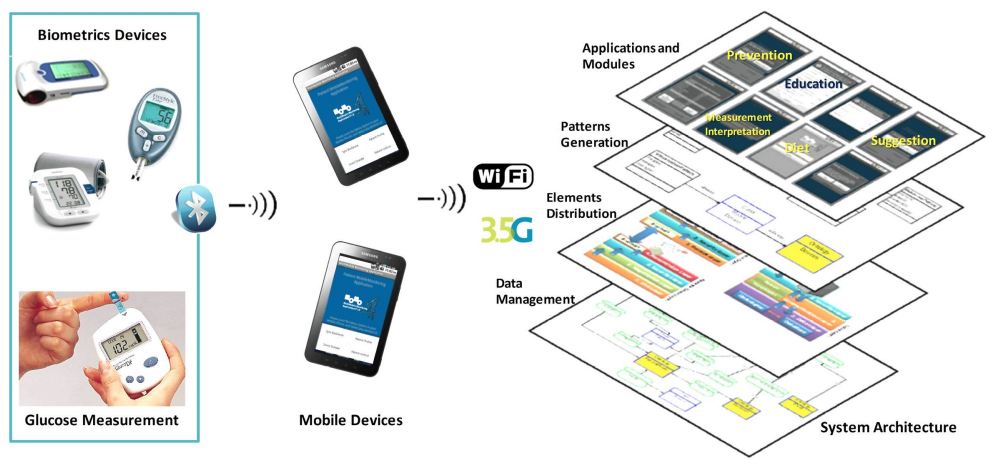

Fig. 2. MoMo platform and relationship between data and device entities 


\subsection{Hybrid Cooperative Reasoner}

As stated in TALISMAN+ framework, a middleware is requested to facilitate context capture from any device, including embedded ones, to program environment's reactivity. TALIS+Engine is the susbsystem were the ontology AMBI2ONT was created to model ambiguity in its two facets, uncertainty and vagueness, together with sensor fusion and reasoning inference engine. Upon uncertainty, the certainty factor (CF) of contextual data is modeled. Vagueness consideration allows model unclearly defined situations like cold room or noise room where different users have different perceptions. Such ontology models places, things in those places such as devices or people, capabilities and linguistic terms. Fig. 3 shows a ContextData individual with a sensor value associated a certainty degree about the credibility of such measure and a set of linguistic terms where each term is associated with a membership function. Thus, in this case the temperature sensor in a given room can be considered mainly hot. Once the measures are modeled considering the uncertainty and the vagueness of the data, semantic inference process is applied, so that implicit data is derived from explicit data. For example, the location associated to each measure is determined, knowing the location of the temperature sensor. Subsequently, a data fusion process is applied which aggregates measures of the same type (e.g. temperature sensor) within the same container (e.g. room). Two different strategies are supported: tourney (the best) and combination, where depending on the scheme applied the best, worst or average measure can be considered. Thus, the temperature values of all sensors within a room are combined.

Finally, the behavior rules defined for an extension of the JFuzzyLogic fuzzy logic engine [13] are executed. Such engine was modified to incorporate to it treatment of both uncertain data and rules. An example of the now supported syntax, which extends JFuzzyLogic engine's Fuzzy control language (FCL), is shown on the bottom left hand side of Fig. 3, where both uncertainty ( $\mathrm{CF} 1$ ) and vagueness (НОT) are considered:
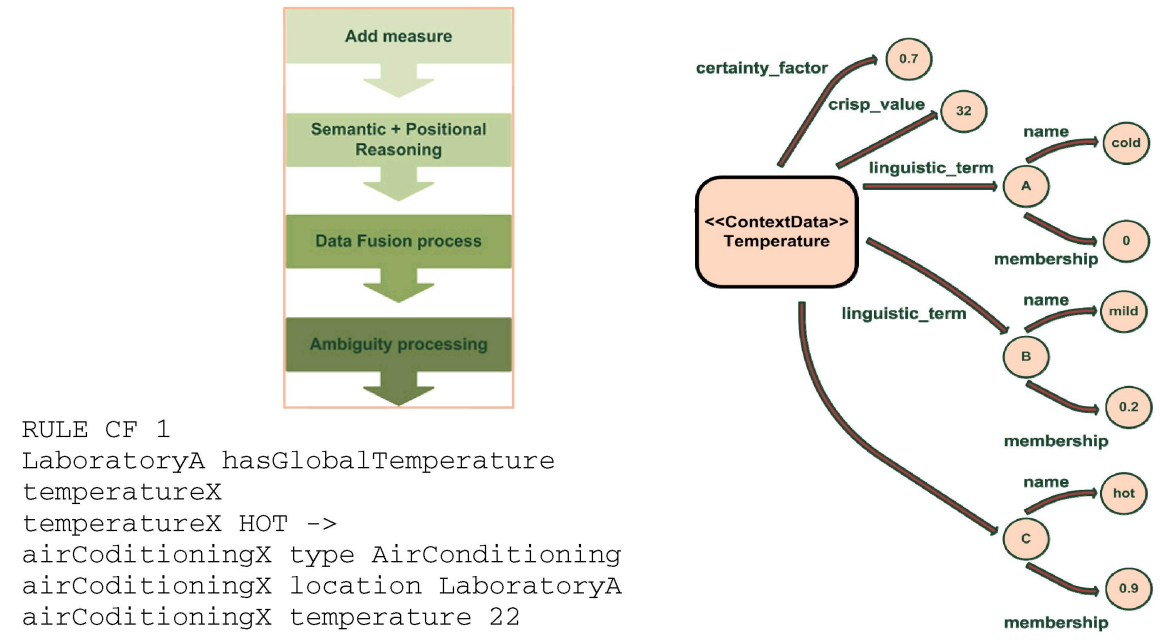

Fig. 3. Hybrid reasoner uncertainty management and AMBI2ONT Ontology 


\subsection{Vision Based Sensor Subsystem}

TALISMAN+ distributed framework relies on private sensoring solutions at the SH to make ad hoc local reasoning to promote healthy and social activities related to active aging. Vision@home is the integrated subsystem that includes a technological infrastructure and vision-based services to monitor and recognize the activity of users at home, including the privacy protection of who perceived with these vision devices. Thus, a double functionality is achieved: dynamically modeling of habitual behavior of people to detect events that may identify abnormal behaviors, and confidential vision-based services to detect and recognize objects and people of interest in the scene and characterization and interpretation of movement to monitor their activity.

Static cameras, pan-tilt, omni-directional and low cost devices RGB-D, like Kinect, were applied to allow better interpretation of motion without forgetting ethical aspects when monitoring people in private spaces. Two cases were addressed to recognize human activity: action recognition using several cameras and action recognition using Kinects. The implemented system recognizes, through cameras, simple actions such as walking, jumping, running and falling. Anonymous people silhouettes are gathered using background removal and next features invariant to scaling and rotation. During recognition, the position of the person is determined at every moment by matching the sequence of postures with the Knowledge Base using Dynamic Time Warping. The system operates at video rate, which is one of the main requirements. Regarding the recognition of actions using Kinects, a classification system was developed from skeletal actions of a person. Thus, Microsoft SDK and OpenNI library functions were tested and positions recognized by Growing Neural Gas. The optimal set of characteristics that increases the accuracy of action recognition algorithm was founded by using evolutionary learning techniques [11]. Fall detection service and other actions are supported by a simple prototype that determines the fall by calculating the hip height.

A context oriented privacy protection model was defined based on levels. Each level defines the nature of the information that will be provided to TALISMAN+ local reasoner or even shown to an authorized caregiver. Each level of representation can depend on the event and the permissions of the stakeholder. Four protection levels were verified as feasible: no alarm, which displays a virtual image of the environment without showing any person; low level alarm, which shows a virtual image of both the environment and the person, showing its location but not its posture; high level alarm, which shows a virtual image of both the environment and the person; and very high level alarm that shows a real image of the environment (Fig. 4).

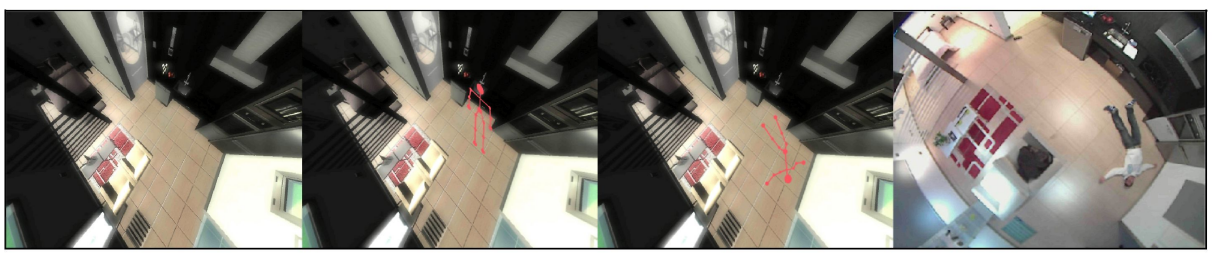

Fig. 4. Vision based privacy degrees depending on the level of alarm 


\subsection{Local Logic Description Reasoner and Security View}

The distributed framework proposed provides functionality in a home environment where critical assurance of privacy is needed for the elderly. Local reasoning should confirm individuals' identities and ensure privacy when they are monitored. Very often, sensors and devices used for these services are perceived as an intrusive element at home. Therefore, privacy and authentication was considered an essential part of TALISMAN+ not just to protect communications but to reinforce users' trust in the use of the news services for active aging and promotion of personal autonomy.

Bearing in mind this user-centered approach, the SH logs all interactions of sensors and monitoring systems that are managing data with the database as well as the data exchanged between the SH and telecare centers. In this way, users with cognitive or physical impairments gain access at any time to the information obtained from the logs, as the system enables tangible and understandable interaction when coupling sensors and actuators into actions. These actions allow users to mentally represent the capabilities of the SH where they perform their daily tasks, regardless of the complexity of the underlying ubiquitous system. The framework implemented a security agent in charge of sending, securing and logging the outward interactions. Fig. 5 shows this agent that receives data from SH and establishes a secure SSL channel between home and telecare entities to provide two-way authentication, non-repudiation, confidentiality and integrity services for the exchange. Previous images processing by the vision based sensor subsystem, this agent sends a virtual hidden image of people at home.

For this purpose it was defined an XML register document that contents the complete sequence of interactions that take place between the server and the smart home as a result of the execution of the service. After ensuring the security of communications for the house with the outside, there is still an important point to solve referred to the way that people at home are identified. Mutual authentication in SSL requires users must be in possession of the corresponding X.509 certificate to operate the system.

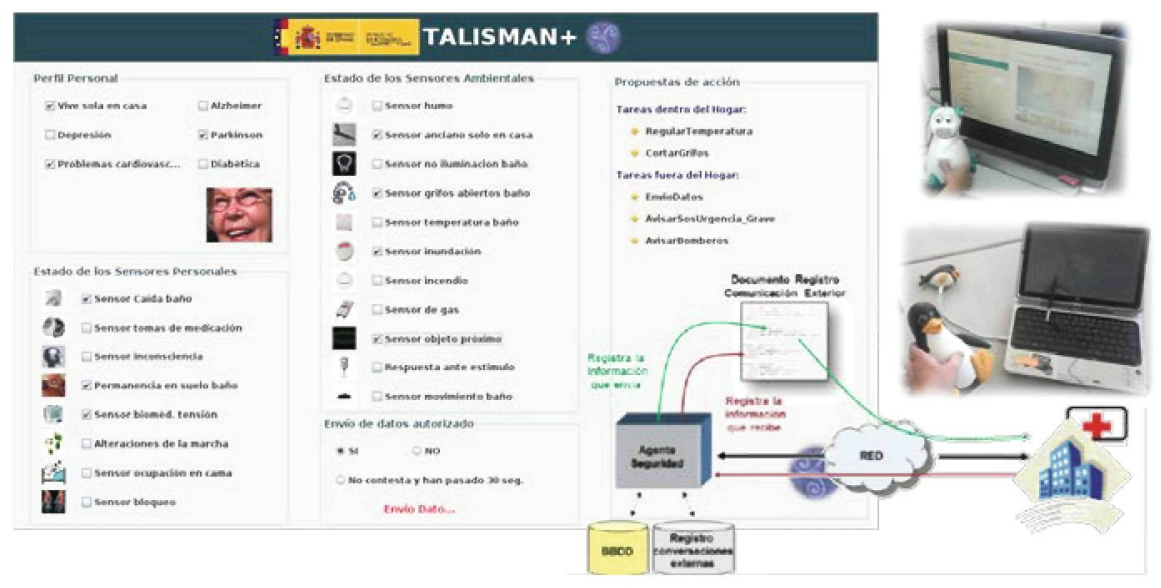

Fig. 5. TALISMAN+ security supporting local reasoning engine 


\section{Conclusions}

The deployment of TALISMAN+ framework at the Accessible Digital Home allows testing a sustainable telecare service with users from Parkinson and Cordial Injury associations. This stage will be initiated shortly once the development and integration stages are completed in order to check reliable reasoning, security and performance.

Acknowledgments. Authors would like to thank the National Plan for Science, Development and Innovation of the Spanish Ministry for Economy and Competitiveness that supported TALISMAN+ (TIN2010-20510) research results detailed in this paper.

\section{References}

1. World Health Organization: Active Ageing. A Policy Framework. Second United Nations World Assembly on Ageing, Madrid, Spain (2002)

2. Cheek, P., Nikpour, L., Nowlin, H.D.: Aging Well With Smart Technology. Nursing Administration Quarterly 29(4), 329-338 (2005)

3. Williams, G., Doughty, K., Bradley, D.A.: A Systems Approach to Achieving CarerNetAn Integrated and Intelligent Telecare System. IEEE Transactions on Information Technology in Biomedicine 2(1), 1-9 (1998)

4. Laiseca, X., Castillejo, E., Orduña, P., Gómez-Goiri, A., López-de-Ipiña, D., González Aguado, E.: Distributed Tracking System for Patients with Cognitive Impairments. In: Bravo, J., Hervás, R., Villarreal, V. (eds.) IWAAL 2011. LNCS, vol. 6693, pp. 49-56. Springer, Heidelberg (2011)

5. Chen, L., Hoey, J., Nugent, C.D., Cook, D.J., Yu, Z.: Sensor-based Activity Recognition. IEEE Transactions on Systems, Man, and Cybernetics, Part C: Applications and Reviews 42(6), 790-808 (2012)

6. Docksteader, L., Benlamri, R.: MORF: A Mobile Health-Monitoring Platform. IT Professional 12(3), 18-25 (2010)

7. Cardinaux, F., Bhowmik, D., Abhayaratne, C., Hawley, M.S.: Video based technology for ambient assisted living: A review of the literature. Journal of Ambient Intelligent Smart Environments 3(3), 253-269 (2011)

8. Islam, K., Shen, W., Wang, X.: Security and Privacy Considerations for Wireless Sensor Networks in Smart Home Environments. In: Proceedings of the IEEE 16th International Conference on Computer Supported Cooperative Work in Design, pp. 626-633 (2012)

9. Gass, O., Maedche, A.: Enabling End-user-driven Data Interoperability - A Design Science Research Project. AMCIS 2011 Proceedings - All Submissions, paper 221 (2011)

10. Villarreal, V., Bravo, J., et al.: Towards Ubiquitous Mobile Monitoring for Health-care and Ambient Assisted Living. In: International Workshop on Ambient Assisted Living, Valencia, Spain (2010)

11. Chaaraoui, A., Climent, P., Flórez, F.: A Review on Vision Techniques applied to Human Behaviour Analysis for Ambient-Assisted Living. Expert Systems with Applications (in press), http://dx.doi.org/10.1016/j.eswa.2012.03.005

12. Balta-Ozkan, N., et al.: Social barriers to the adoption of smart homes. Energy Policy (2013), http://dx.doi.org/10.1016/j.enpol.2013.08.043

13. Cingolani, P., Alcalá-Fdez, J.: jFuzzyLogic: a Java Library to Design Fuzzy Logic Controllers According to the Standard for Fuzzy Control Programming. International Journal of Computational Intelligence Systems 6 (supp. 1) (2013) 